\title{
La irrupción de las tecnologías de la información y la comunicación (TIC), un reto en la gestión de las competencias digitales de los profesores universitarios en el Ecuador
}

\author{
Cejas Martínez Magda Franciscaㄹ, Lozada Arias Brenda Nohemi², \\ Urrego Ana Jacqueline ${ }^{3}$, Mendoza Velazco Derling José ${ }^{4}$, Rivas Urrego Gabriela 5 \\ magda.cejas@unach.edu.ec, brenda.lozada.uc@gmail.com, \\ anaurrego7o@gmail.com, derling969@gmail.com, gabrielarivasu@gmail.com
}

${ }^{1}$ Profesora investigadora de la Universidad Nacional de Chimborazo, UNACH y Universidad Nacional de las Fuerzas Armadas ESPE, Riobamba, 060150, Ecuador.

${ }^{2}$ Universidad de Carabobo UC, Valencia, 2005, Carabobo, Venezuela.

${ }_{3}^{3}$ Universidad Iberoamericana del Ecuador UNIB.E, Quito, 170523, Ecuador

${ }^{4}$ Universidad Nacional de Educación, UNAE. Chuquipata, El Cañar, 030154, Ecuador

${ }^{5}$ Universidad Pedagógica Experimental Libertador UPEL, Caracas, 1040, Venezuela

DOI: 10.17013/risti.37.131-148

\begin{abstract}
Resumen: El estudio tiene como objetivo evaluar las competencias digitales de los profesores universitarios en el Ecuador durante el lapso académico 2019-2020 en la Universidad Nacional de Educacion UNAE durante el ciclo académico 20192020 en Ecuador. La investigación parte de un enfoque mixto y un diseño de campo descriptivo-explicativo, a través de una muestra estratificada no probabilística de 37 docentes. Se utilizó la guía de observación directa de los participantes y la aplicación de dos cuestionarios. La presente investigación concluye que la mayoría de los profesores utilizan las TIC sólo para la información y la comunicación, existiendo disponibilidad de acceso a la tecnología por parte de la institución, pero una alta deficiencia en la capacitación de investigación de los mismos.
\end{abstract}

Palabras-clave: profesor; universidad; tecnología; internet; educación superior.

The irruption of information and communication technologies (ICT), a challenge in the management of digital competences of University Professors in Ecuador

Abstract: The study aims to evaluate the digital competencies of university teachers in Ecuador during the 2019-2020 academic year at the National University of Education (UNAE) during the 2019-2020 academic year in Ecuador. The research is based on a mixed approach and a descriptive-explicative field design, through a stratified non-probabilistic sample of 37 teachers. The guide of direct observation 
of the participants and the application of two questionnaires were used. The present research concludes that the majority of teachers use ICTs only for information and communication, there being availability of access to technology on the part of the institution, but a high deficiency in the research training of teachers.

Keywords: professor, university, technology, internet, education university.

\section{Introducción}

Para llevar a cabo sus funciones, el actual profesor universitario tiene varios retos en el proceso de enseñanza-aprendizaje. Cada día el desarrollo de competencias para gestionar el aula es más exigente, la irrupción de las Tecnologías de la Información y la Comunicación (en adelante por su sigla TIC), en muchos aspectos, ha servido de apoyo para fortalecer las competencias, y en otros casos de difícil manejo y adaptación según el profesor, el contexto y el entorno de aprendizaje (Makewa, Ngussa y Kuboja, 2018).

Este contexto de adaptación y generación de competencias, unido a un sentido de responsabilidad que reconoce la trascendencia, la dirección de las acciones y el impacto que éstas pueden tener en cada estudiante, lleva a establecer mecanismos y estrategias de enseñanza-aprendizaje coherentes con las necesidades de todos los actores vinculados al proceso educativo de los tiempos actuales (Lozada, 2019). Se pueden establecer diferencias significativas entre el profesor que gestiona y practica técnicas o estrategias de enseñanza con apoyo en las TIC, un profesor que se resiste a hacerlo, que no las conoce ni las aplica.

En consecuencia, las competencias de un profesor universitario se conciben como competencias integrales y alineadas a las exigencias de los tiempos que les corresponden para impartir el conocimiento. Esta era se caracteriza particularmente por el manejo dinámico y rápido de la información, donde la tecnología constituye una de las transformaciones más vertiginosas y cambiantes que han existido en las últimas décadas (Battro y Percival, 2017).

Es ineludible pensar que el factor tecnológico y de las comunicaciones beneficia a las sociedades en muchos aspectos, pero del mismo modo, el uso inadecuado interfiere en otros aspectos de naturaleza personal y de comportamiento, en particular el de la concentración, donde muchos afirman que es una interferencia en el desarrollo de la inteligencia, una de las razones de la disponibilidad instantánea de la información (Makewa, Ngussa y Kuboja, 2018) por eso es importante conocer la forma en que los profesores se apropian de las TIC en su práctica educativa, ya que hay otros críticos que establecen que los recursos tecnológicos pueden ser utilizados para mejorarla. La irrupción de las nuevas tecnologías nos obliga a educar de una manera diferente, el papel de los profesores debe ser el de guía en la educación de cada alumno, no el de fuente de toda la información y todo el conocimiento, como ocurría hasta ahora, y la Tecnología puede ayudar a motivar en el proceso de aprendizaje (Gardner, 2011).

De manera introductoria y a la luz de lo anterior, la investigación que se presenta parte de una descripción del contexto en el que se establecen las diferentes variables que intervienen en el estudio, luego se describe el proceso metodológico, donde se explica el objetivo, el paradigma, el tipo y las técnicas de investigación utilizadas, continuando con 
la disertación a través del análisis de los resultados, para terminar con las conclusiones. El estudio es parte de un estudio piloto realizado en la Universidad Nacional de Educación (UNAE), ubicada en la ciudad de Azogues, Ecuador.

\section{Contexto teórico}

Los desafíos que condicionan esta era globalizada y tecnológica, a nivel mundial han incorporado sustancialmente elementos que cambian e influyen de manera rápida y abrumadora con respecto a la forma en que concebimos el mundo. Desde los procesadores de datos, la tecnología tridimensional de alta definición hasta las aplicaciones y los teléfonos móviles cercanos a una computadora, todos constituyen mecanismos que se han incorporado a cada una de las actividades diarias y cotidianas en los hogares, los trabajos, las escuelas y las universidades.

Teniendo en cuenta lo anterior, es inminente la conciliación que la educación y las competencias de los profesores universitarios requieren ante los nuevos paradigmas tecnológicos a los que deben adaptarse. En la dirección de lo que algunos especialistas en la materia expresaron como Pedró (2016) afirma que las competencias profesionales de los profesores, y las facilidades e incentivos para su continuo desarrollo, son la clave. Pérez (2013) expresa que estas competencias son óptimas, y es evidente que el recurso destinado a la tecnología mejora la calidad de los procesos de aprendizaje y cuando no se dan estas condiciones, la irrupción de la tecnología se traduce en nuevos problemas para los profesores en lugar de proponer soluciones que optimicen su esfuerzo.

En América Latina se realiza un esfuerzo continuo por la disponibilidad y el acceso a las tecnologías, a lo que se suman brechas muy amplias a nivel social, cultural, económico, educativo y de inclusión. Algunos países más que otros muestran niveles diferentes, una de las brechas más significativas es la educación. En este sentido Battro y Percival, (2017), indican que "no hay dudas sobre el impacto del fenómeno de la globalización en las relaciones y transacciones humanas de todo tipo, pero hay que reconocer que, paradójicamente, hasta el momento, el principal sector que ha resistido frontalmente y con tenacidad a esta globalización es la propia educación” (p.15). Los actores que participan activamente en el proceso educativo universitario deben hacer hincapié en la adaptación y mejora de las condiciones del entorno tecnológico para que sea posible llevar a cabo un proceso de enseñanza-aprendizaje acorde con los tiempos actuales.

Por otra parte, aun cuando esta realidad es vista y reconocida por el ámbito educativo y social, entre otros, los estudiantes universitarios con una edad promedio de 16 a 25 años, la mayoría de ellos tienen conocimientos y habilidades para el uso de las TIC, en todos aquellos asociados con las redes sociales, para Chiecher y Lorenzati, (2017), "Si hay un rasgo que caracteriza a los adolescentes y jóvenes de hoy en día es el hecho de que nacieron en un mundo tecnológico, que se mueve a través de las tecnologías" (p.262), en efecto, las tecnologías actuales permiten transformar los entornos educativos tradicionales y contribuyen a la generación de nuevos entornos.

Sin embargo, un estudio realizado durante los últimos cinco años por la Unesco (2013) muestra que aproximadamente el 50\% de la población entre 5 y 19 años de edad en los países de América Latina se encuentra fuera de los sistemas educativos formales y con una reparación que no les permite integrarse plenamente en la economía moderna e incluso 
dejándolos en riesgo de formar parte de los segmentos de población que permanecen por debajo de la línea de pobreza, esta condición intensifica la generación de modelos de crecimiento del subempleo, aumentando el desempleo juvenil y el trabajo precario por la falta de preparación profesional. De igual manera, los profesores universitarios entre 45 y 65 años muestran severas dificultades para relacionarse con estas tecnologías dentro y fuera del aula, muchos de ellos no las utilizarían si no estuvieran obligados por los diferentes sistemas que las universidades han diseñado para el uso e inclusión de las TIC en las clases (Mendoza, et al., 2019a).

En el mismo orden de ideas, si se asume al estudiante como receptor en el proceso educativo, debe considerarse que actualmente las expectativas de los estudiantes respecto de la realidad digitalizada, debido a la fuerte influencia que las tecnologías tienen en los más jóvenes y la forma en que conciben la realidad y lo que ello implica para motivar su aprendizaje, De Llano (2013) manifiesta un ejemplo de ello, donde lo denota un informe realizado por profesores de la Universidad Católica Andrés Bello en Venezuela, a través de un estudio se encontró que el 95\% de los estudiantes universitarios tienen una computadora en casa, el 73\% un smartphone. El 98\% está conectado a Facebook y el 73\% utiliza redes sociales como Youtube, Twitter, Google, Instagram entre otros diariamente. En la literatura, esta generación ha sido llamada la Generación NET, los nativos digitales, los milenarios o incluso los superficiales, lo que denota la tendencia a desarrollar habilidades y prácticas profesionales y sociales emergentes que es necesario apreciar en esta nueva generación, que es diferente y se adapta al nuevo mundo de la globalización y el desarrollo de las TIC, destacando que la generación NET no se identifica necesariamente por ser más o menos creativa que las generaciones anteriores (Mendoza et al., 2019b).

Para Spante et al., (2018) esta generación ha estado expuesta a un mundo tecnológico radicalmente distinto al del texto impreso, paradójicamente al que estuvieron expuestas las generaciones anteriores, de donde proceden la mayoría de los profesores universitarios activos en estos tiempos. Como se ha destacado esta realidad interconectada no es un fenómeno tecnológico, sino un nuevo entorno cultural caracterizado por la interconexión, la interactividad, la ubicuidad de la tecnología, la libertad de acción, la valoración de la innovación y la no linealidad como estructura fundamental.

En el Ecuador, en particular, se observa la necesidad de contar con estrategias educativas eficaces que permitan a los maestros mantener los niveles de concentración y de atención y alcanzar el objetivo de aprender a través de los medios disponibles, gestionando adecuadamente su uso. Para ello, debe estar dotado de habilidades básicas y técnicas que le permitan estar a la vanguardia del conocimiento de las TICs, Web 1.0 y Web 2.0

Es necesario considerar que la labor del docente en el aula en los tiempos actuales, fragmenta algunos paradigmas educativos tradicionales, y como líder del proceso, le corresponde enfrentar las consecuencias de las prácticas del uso de los diferentes dispositivos que se han convertido en parte importante para la comunicación de todos, según Gardner, (2011) la irrupción de las nuevas tecnologías nos obliga a educar de una manera diferente, la cuestión es descubrir cómo aprende una persona, descubrir sus pasiones, que son muy importantes, y utilizar todos los recursos humanos y tecnológicos que nos ayuden. 
Para Zuñiga, (2016) "Las competencias digitales son un elemento que contribuye significativamente al desarrollo del autoaprendizaje y, en gran medida, son también herramientas que permiten aumentar el capital de conocimiento científico, promoviendo el uso efectivo y eficiente de los recursos digitales" (p.12). La gestión de las competencias tecnológicas o digitales en los profesores universitarios es una necesidad y también un reto, ya que debe haber disponibilidad para que adquieran o desarrollen esas habilidades y las practiquen en las clases de manera efectiva.

En vista de las consideraciones expresadas, los autores se vieron motivados a realizar un estudio piloto sobre el estado de la irrupción de las tecnologías de la información y la comunicación (TIC), como un desafío en la gestión de las competencias de los profesores universitarios en el Ecuador. Con la intención científica primaria de evidenciar las capacidades y habilidades tecnológicas y la aplicabilidad en el aula de la muestra de profesores del estudio en la UNAE, para ello fue necesario enmarcar la investigación en una metodología que permitiera obtener datos e información efectiva de la realidad método de estudio recomendado por Cejas et al., (2019).

\subsection{Objetivos de la investigación}

El estudio tiene como objetivo evaluar las competencias digitales de los profesores universitarios en el Ecuador durante el lapso académico 2019-2020 en la Universidad nacional de Educacion UNAE.

\section{Metodología}

El estudio se enmarcó en una investigación de enfoque mixto, si bien es cierto los datos se extrajeron en base a su diseño de campo, el soporte teórico y la revisión documental especializada permitieron complementar, definir y describir el objeto de estudio y medirlo en el tiempo estipulado para ello, sin tener que influir en las variables involucradas, lo que implica que fue descriptivo-explicativo (Yanez et al., 2019).

\subsection{Población y muestra}

El objetivo general fue estudiar el surgimiento de las tecnologías de la información y la comunicación (TIC), como un desafío en el desarrollo de la gestión de las competencias digitales de los docentes universitarios, a partir de un estudio de campo en el período 2018-2019, en la Universidad Nacional de Educacion UNAE. La población docente de la UNAE es de 250 profesores activos. Para la muestra se estableció un muestreo aleatorio estratificado no probabilístico, con un total de 37 profesores (ver tabla 1).

\begin{tabular}{llll}
\hline Estrato & Población & Proporción & Muestra proporcional \\
\hline 1/Docentes contratados & 180 & $72 \%$ & 26 \\
\hline 2/Docentes titulares & 70 & $28 \%$ & 11 \\
\hline Total & 250 & $100 \%$ & 37 \\
\hline
\end{tabular}

Tabla 1 - Distribución de la muestra aleatoria estratificada de tipo no probabilística en la investigación 
Se determinó la relación estratificada predeterminada que existe en la universidad UNAE donde 180 docentes laboran como contratados ocasionales, con una proporción de $72 \%$ de la población, como también 70 docentes titulares que representan un $28 \%$ del total. En base a esta proporción 70/30 de forma aleatoria no probabilista se aplicaron invitaciones de tipo participativa a los docentes contratados y titulares a formar parte del estudio, estableciendo una muestra de 26 docentes contratados y 11 docentes titulares para una muestra final de 37 profesores método recomendado por Wright, Noble y Bailer, (2007).

\subsection{Instrumentos de recolección de datos}

Como instrumento para la recolección de información de carácter cualitativo, se diseñó una guía de observación utilizada por los investigadores para observar la realidad durante las sesiones de clase de los profesores participantes (ver tabla 2).

\begin{tabular}{ll}
\hline \multicolumn{1}{c}{ Guía de observación } \\
\hline Universidad Nacional de Educacion UNAE & Fecha:______ \\
\hline Nombre del Investigador: & \\
\hline $\begin{array}{l}\text { Tipo de actividad } \\
\text { educativa }\end{array}$ & \\
\hline $\begin{array}{l}\text { Tipo de herramienta } \\
\text { tecnológica aplicada } \\
\text { en el aula }\end{array}$ \\
\hline $\begin{array}{l}\text { Herramienta } \\
\text { de búsqueda e } \\
\text { investigación. }\end{array}$ \\
\hline $\begin{array}{l}\text { Software operativo de } \\
\text { navegación }\end{array}$ \\
\hline Presenta dominio \\
tecnológico \\
\hline Observaciones:
\end{tabular}

Tabla 2 - Guía de Observación aplicada por los investigadores a los profesores de la Universidad Nacional de Educacion UNAE.

Como segundo instrumento de recolección de datos, se diseñaron dos cuestionarios basado en la escala de Likert con cuatro opciones de respuesta. Como primera opción "nulo" con un valor numérico de puntaje 1, luego la opción "básico" con un valor escalar de 2 puntos. Como tercera opción "intermedio", con un valor escalar de 3 puntos, seguidamente la opción "avanzado" con 4 puntos, finalmente la opción "muy avanzado" con el puntaje mayor de 5 puntos (ver tabla 3 ).

La opción aplicada a la muestra total de profesores involucrados en el estudio, derivado del trabajo de investigación de Zuñiga, (2016), sobre las dimensiones, factores y categorías de las habilidades digitales en los profesores universitarios. 


\section{Cuestionario de Investigación / Web1.o}

\begin{tabular}{|c|c|c|c|c|c|c|}
\hline \multicolumn{4}{|c|}{ Universidad nacional de Educacion UNAE } & \multirow{2}{*}{\multicolumn{3}{|c|}{ Fecha: }} \\
\hline \multicolumn{2}{|c|}{ Pregunta: } & \multicolumn{2}{|c|}{ Opciones } & & & \\
\hline$N^{o}$ & $\begin{array}{l}\text { De los software Web 1.o que } \\
\text { se presentan a continuación, } \\
\text { indique cuál es su nivel de } \\
\text { dominio. }\end{array}$ & Nulo & Básico & Intermedio & Avanzado & $\begin{array}{c}\text { Muy } \\
\text { avanzado }\end{array}$ \\
\hline 1 & Uso de Microsoft Office Word & & & & & \\
\hline 2 & $\begin{array}{l}\text { Uso de Microsoft Office } \\
\text { PowerPoint }\end{array}$ & & & & & \\
\hline 3 & Uso de Microsoft Office Excel & & & & & \\
\hline 4 & $\begin{array}{l}\text { Uso de Microsoft Office } \\
\text { Publisher }\end{array}$ & & & & & \\
\hline 5 & Uso de Writer's & & & & & \\
\hline 6 & $\begin{array}{l}\text { Elaboración de base de datos } \\
\text { Access }\end{array}$ & & & & & \\
\hline 7 & $\begin{array}{l}\text { Edición de imágenes y } \\
\text { gráficos en Draw, Photoshop } \\
\text { o Paint }\end{array}$ & & & & & \\
\hline 8 & Uso de SSPS & & & & & \\
\hline 9 & Uso de Digital Library & & & & & \\
\hline 10 & $\begin{array}{l}\text { Búsqueda en sistemas Google. } \\
\text { com, Yahoo.com, Firefox, Big }\end{array}$ & & & & & \\
\hline 11 & Uso de Google-Docs & & & & & \\
\hline 12 & $\begin{array}{l}\text { Uso de correo Hotmail, Gmail } \\
\text { y Yahoo Mail }\end{array}$ & & & & & \\
\hline
\end{tabular}

Tabla 3 - Cuestionario aplicado a los docentes participantes para el análisis de las competencias y dominio web1.o

Como segundo instrumento para el análisis de las competencias digitales y tecnológicas de los profesores de aula en base al web2.o se aplicó el siguiente cuestionario (ver tabla 4).

\begin{tabular}{|c|c|c|c|c|c|c|}
\hline \multicolumn{7}{|c|}{ Cuestionario de Investigación / Web 2.o } \\
\hline \multicolumn{4}{|c|}{ Universidad nacional de Educacion UNAE } & Fecha: & I & \\
\hline \multicolumn{2}{|c|}{ Pregunta: } & \multicolumn{2}{|c|}{ Opciones } & & & \\
\hline$N^{o}$ & $\begin{array}{l}\text { De los software Web 2.o que } \\
\text { se presentan a continuación, } \\
\text { indique cuál es su nivel de } \\
\text { dominio. }\end{array}$ & Nulo & Básico & Intermedio & Avanzado & $\begin{array}{c}\text { Muy } \\
\text { avanzado }\end{array}$ \\
\hline 1 & Los editores de video & & & & & \\
\hline 2 & $\begin{array}{l}\text { Gestión de la Plataforma } \\
\text { Educativa: Moodle, Edmodo }\end{array}$ & & & & & \\
\hline
\end{tabular}




\begin{tabular}{ll}
\hline 3 & $\begin{array}{l}\text { Video Chat y Aulas Virtuales, } \\
\text { Messenger, Skype, Zoom, } \\
\text { Microsoft-Teams }\end{array}$ \\
\hline $4 \quad$ & $\begin{array}{l}\text { Actividad educativa en las } \\
\text { redes sociales Facebook, } \\
\text { Twitter, Instagram }\end{array}$ \\
\hline $5 \quad$ & $\begin{array}{l}\text { Gestión del Blog Educativo: } \\
\text { Blogger, WordPress }\end{array}$ \\
\hline 6 & $\begin{array}{l}\text { Videoconferencia a través de } \\
\text { Skype, Zoom, Adobe Connect }\end{array}$ \\
\hline 8 & $\begin{array}{l}\text { Manejo de herramientas de } \\
\text { trabajo en grupo } \\
\text { (Google-Docs) }\end{array}$ \\
\hline 9 & $\begin{array}{l}\text { Elaboración y edición de } \\
\text { páginas web: Google-Sites, }\end{array}$ \\
\hline
\end{tabular}

Tabla 4 - Cuestionario aplicado a los docentes participantes para el análisis de las competencias y dominio web 2.0

\subsection{Confiabilidad}

Para establecer la confiabilidad de los instrumentos de recolección de datos cuantitativos (Tabla 3) en la investigación, se aplicó una prueba piloto designada a 10 docentes. Determinándose un reproducibilidad o consistencia de las mediciones a través del coeficiente alfa de Cronbach con un nivel de confianza de o,890 para el cuestionario de análisis web1.o, considerado un valor alto según Mendoza et al., (2018) (ver tabla 5).

\begin{tabular}{ccc}
\hline & \multicolumn{2}{c}{ Confiabilidad } \\
\hline Alfa Cronbach & $\begin{array}{c}\text { Alfa Cronbach basado en } \\
\text { elementos estandarizados }\end{array}$ & N de elementos (items) \\
\hline 0.890 & 0.887 & 12 \\
\hline
\end{tabular}

Tabla 5 - Resultado del coeficiente estadístico alfa de cronbach aplicado al cuestionario para el análisis de las competencias y dominio web 1.0

De igual manera, se aplicó el coeficiente estadístico alfa de Cronbach al cuestionario de recolección de datos web 2.0 (tabla 4), con un resultado de 0,859 considerado un valor alta confiabilidad según los autores previamente citados (ver tabla 6).

\begin{tabular}{ccc}
\hline \multicolumn{3}{c}{ Confiabilidad } \\
\hline Alfa Cronbach & $\begin{array}{c}\text { Alfa Cronbach basado en } \\
\text { elementos estandarizados }\end{array}$ & N elementos (items) \\
\hline 0.859 & 0.857 & 9 \\
\hline
\end{tabular}

Tabla 6 - Resultado del coeficiente estadístico alfa de cronbach aplicado al cuestionario para el análisis de las competencias y dominio web 2.0 


\subsection{Análisis de los resultados}

Para el análisis de los resultados, se aplicó la triangulación de datos o contrastación de resultados. Los datos obtenidos por la guía de observación y sustentos teóricos fueron analizados de forma interpretativa por los autores del estudio. Los datos cuantitativos de los cuestionarios, fueron analizados de forma descriptiva porcentual mediante la frecuencia y consideración del nivel de dominio de los profesores encuestados (Rendón, Villasís y Miranda, 2016).

La triangulación se refiere al uso de múltiples métodos o fuentes de datos en la investigación para desarrollar una comprensión integral de los fenómenos (Agnes y Norwich, 2007). La triangulación o contrastación de los resultados emerge al unificar de forma descriptiva los resultados porcentuales o cuantitativos del estudio con los datos cualitativos interpretativos emergentes (Azulai y Rankin, 2012).

Los datos cualitativos fueron postergados en Google Drive Suite y los datos cuantitativos analizados mediante el sosftware, Statistical Package for the Social Sciences (SPSS) versión 25. Los resultados se presentaron en tablas de frecuencia y gráficos multivariados, para ofrecer su comprensión de acuerdo con los hallazgos encontrados en el desarrollo de la investigación.

\section{Resultados y discusión}

Se realizó una revisión teórica de las investigaciones apoyadas en el Ecuador, que permitió a los autores tener una referencia del estado de las competencias digitales de los profesores universitarios en otras áreas de la educación superior, lo que podría complementar el estudio de campo sobre la irrupción de las tecnologías de la información y la comunicación (TIC), como un desafío en la gestión de las competencias de los profesores universitarios en la UNAE en el período 2019-2020.

Entre los hallazgos encontrados, destacamos las coincidencias en cuanto a la gestión de las competencias digitales y tecnológicas de los profesores de aula, y el desafío que representa para la mayoría al incorporar la variedad de herramientas que ofrecen las TIC hoy en día, considerando la Web 1.o y la Web 2.o. A través de la lista de verificación y de la observación directa de los participantes, los profesores estudiados demostraron sus capacidades, obteniendo los siguientes resultados que se presentan en la tabla 7.

\begin{tabular}{|c|c|c|c|c|c|c|c|c|c|c|c|c|}
\hline \multirow[t]{2}{*}{ - } & \multirow{2}{*}{\multicolumn{2}{|c|}{ Nulo }} & \multicolumn{2}{|c|}{ Básico } & \multicolumn{2}{|c|}{ Intermedio } & \multicolumn{2}{|c|}{ Avanzado } & \multirow[t]{2}{*}{$\underset{\text { Muy }}{\text { avanzado }}$} & \multicolumn{2}{|c|}{$\begin{array}{l}\text { Usar como } \\
\text { apoyo en } \\
\text { las clases }\end{array}$} & \multirow{2}{*}{ 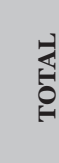 } \\
\hline & & & & & & & ro & esores & & & & \\
\hline $\begin{array}{l}\text { Uso de Microsoft } \\
\text { Office Word }\end{array}$ & o & - & 25 & $67,5 \%$ & 10 & $27,0 \%$ & 2 & $5,4 \%$ & - & 22 & $59,4 \%$ & 37 \\
\hline $\begin{array}{l}\text { Uso de } \\
\text { Microsoft Office } \\
\text { PowerPoint }\end{array}$ & O & - & 20 & $54,0 \%$ & 13 & $35,1 \%$ & 4 & $10,8 \%$ & - & 37 & $100 \%$ & 37 \\
\hline
\end{tabular}




\begin{tabular}{|c|c|c|c|c|c|c|c|c|c|c|c|c|}
\hline $\begin{array}{l}\text { Uso de Microsoft } \\
\text { Office Excel }\end{array}$ & o & - & 20 & $54,0 \%$ & 16 & $43,2 \%$ & 1 & $2,7 \%$ & - & 37 & $100 \%$ & 37 \\
\hline $\begin{array}{l}\text { Uso de Microsoft } \\
\text { Office Publisher }\end{array}$ & 21 & $56,7 \%$ & 8 & $21,6 \%$ & 5 & $13,5 \%$ & 3 & $8,1 \%$ & - & 12 & $32,4 \%$ & 37 \\
\hline Uso de Writer's & 30 & $81,0 \%$ & 4 & $10,8 \%$ & 3 & $8,1 \%$ & o & - & - & o & - & 37 \\
\hline $\begin{array}{l}\text { Elaboración de } \\
\text { base de datos } \\
\text { Access }\end{array}$ & 32 & $86,4 \%$ & 5 & $13,5 \%$ & o & - & $\mathrm{o}$ & - & - & 3 & $8,1 \%$ & 37 \\
\hline $\begin{array}{l}\text { Edición de } \\
\text { imágenes } \\
\text { y gráficos } \\
\text { en Draw, } \\
\text { Photoshop, Paint }\end{array}$ & 30 & $81,0 \%$ & 5 & $13,5 \%$ & 2 & $5,4 \%$ & $\mathrm{O}$ & - & - & 7 & $18,9 \%$ & 37 \\
\hline Uso de SSPS & 31 & $83,7 \%$ & 4 & $10,8 \%$ & 2 & $5,4 \%$ & o & - & - & 3 & $8,11 \%$ & 37 \\
\hline $\begin{array}{l}\text { Uso de Digital } \\
\text { Library }\end{array}$ & 22 & $59,4 \%$ & 8 & $21,6 \%$ & 4 & $10,8 \%$ & 3 & $8,1 \%$ & - & 15 & $40,5 \%$ & 37 \\
\hline $\begin{array}{l}\text { Búsqueda en: } \\
\text { Google.com, } \\
\text { Yahoo.com } \\
\text { Firefox, Big }\end{array}$ & o & - & 31 & $83,7 \%$ & 6 & $16,2 \%$ & o & - & - & 37 & $100 \%$ & 37 \\
\hline $\begin{array}{l}\text { Uso de Google- } \\
\text { Docs }\end{array}$ & 25 & $67,5 \%$ & 6 & $16,2 \%$ & 5 & $13,5 \%$ & 1 & $2,7 \%$ & - & 7 & $18,9 \%$ & 37 \\
\hline $\begin{array}{l}\text { Uso de correo } \\
\text { Hotmail, Gmail, } \\
\text { Yahoo! mail }\end{array}$ & o & - & 31 & $83,7 \%$ & 5 & $13,5 \%$ & 1 & $2,7 \%$ & - & 37 & $100 \%$ & 37 \\
\hline $\begin{array}{l}\text { Promedio total } \\
\text { de herramientas } \\
\text { WEB } 1.0\end{array}$ & & $43,0 \%$ & & $37,6 \%$ & & $15,9 \%$ & & $3,3 \%$ & o\% & & $48,8 \%$ & $100 \%$ \\
\hline
\end{tabular}

Tabla 7 - Dominio de las herramientas TIC, Web 1.o por parte de los docentes de la UNAE, Ecuador, período 2019-2020.

En cuanto al dominio de las herramientas tecnológicas o TIC de la Web 1.0 (ver tabla 7), los resultados obtenidos en 37 profesores seleccionados en el estudio, representaron el $56,76 \%$, indicando que no dominaban las herramientas de Microsoft Office Publisher, el 81,08\% no dominaban el uso del procesador de textos Writer, 86. El 49\% no sabía crear una base de datos con Access, el 81,08\% no dominaba la edición de imágenes y gráficos en Draw, Photoshop y Paint, el 83,78\% no conocía el manejo de herramientas estadísticas por SSPS, el 59,46\% no dominaba el manejo de Bibliotecas digitales y el $67,57 \%$ no dominaba las herramientas de trabajo de Google - Docs. De igual forma, ninguno de los participantes se calificó con un nivel muy avanzado en el dominio de las TIC.

Sin embargo, el 67,57\% reconocía el dominio básico del manejo de las herramientas de Microsoft Office Word, el 54,05\% el uso de las herramientas de Microsoft Office 
PowerPoint y Excel, y el 83,78\% el dominio de los navegadores de búsqueda como Google.com, Yahoo.com, Firefox y Big, administrar correos electrónicos como Hotmail, Gmail, Yahoo! mail. El promedio de dominio de las 12 herramientas tecnológicas de la web 1.0 es Nulo en $43.02 \%$ y básico en $37.61 \%$.

\begin{tabular}{|c|c|c|c|c|c|c|c|c|c|c|c|c|}
\hline \multirow{2}{*}{ Los editores de video } & \multicolumn{2}{|c|}{ Nulo } & \multicolumn{2}{|c|}{ Básico } & \multicolumn{2}{|c|}{ Intermedio } & \multirow{2}{*}{\multicolumn{2}{|c|}{$\begin{array}{l}\text { Avanzado } \\
\text { rofesores }\end{array}$}} & \multirow{3}{*}{$\begin{array}{c}\begin{array}{c}\text { Muy } \\
\text { avanzado }\end{array} \\
-\end{array}$} & \multicolumn{2}{|c|}{$\begin{array}{l}\text { Usar como } \\
\text { apoyo en } \\
\text { las clases }\end{array}$} & \multirow{3}{*}{ 离 } \\
\hline & & & & & & & & & & & & \\
\hline & 30 & $81,0 \%$ & 4 & $10,8 \%$ & 2 & $5,4 \%$ & 1 & $2,7 \%$ & & 2 & $5,4 \%$ & \\
\hline $\begin{array}{l}\text { Gestión de la } \\
\text { Plataforma } \\
\text { Educativa: Moodle, } \\
\text { Edmodo }\end{array}$ & o & - & 15 & $40,5 \%$ & 17 & $45,9 \%$ & 5 & $13,5 \%$ & - & 27 & $72,9 \%$ & 37 \\
\hline $\begin{array}{l}\text { Video Chat y Aulas } \\
\text { Virtuales, Messenger, } \\
\text { Skype, Zoom }\end{array}$ & 5 & $13,5 \%$ & 17 & $45,9 \%$ & 10 & $27,0 \%$ & 5 & $13,5 \%$ & - & 15 & $40,5 \%$ & 37 \\
\hline $\begin{array}{l}\text { Actividad educativa } \\
\text { en las redes sociales } \\
\text { Facebook, Twitter, } \\
\text { Instagram }\end{array}$ & 25 & $67,5 \%$ & 10 & $27,0 \%$ & 2 & $5,4 \%$ & 0 & - & - & 12 & $32,4 \%$ & 37 \\
\hline $\begin{array}{l}\text { Gestión del Blog } \\
\text { Educativo: Blogger, } \\
\text { Wordpress }\end{array}$ & 21 & $56,7 \%$ & 11 & $29,73 \%$ & 3 & $8,11 \%$ & 2 & $5,41 \%$ & - & 9 & $24,3 \%$ & 37 \\
\hline $\begin{array}{l}\text { Videoconferencia } \\
\text { a través de Skype, } \\
\text { Zoom, Adobe Connect }\end{array}$ & 21 & $56,7 \%$ & 10 & $27,0 \%$ & 5 & $13,5 \%$ & 1 & $2,70 \%$ & - & 5 & $13,5 \%$ & 37 \\
\hline $\begin{array}{l}\text { Manejo de } \\
\text { herramientas de } \\
\text { trabajo en grupo } \\
\text { (Google-Docs) }\end{array}$ & 22 & $59,4 \%$ & 8 & $21,6 \%$ & 6 & $16,2 \%$ & 1 & $2,7 \%$ & - & 1 & $2,7 \%$ & 37 \\
\hline $\begin{array}{l}\text { Elaboración y edición } \\
\text { de páginas web: } \\
\text { Google-Sites, Wix }\end{array}$ & 35 & $94,5 \%$ & 2 & $5,4 \%$ & o & - & $\mathrm{o}$ & - & - & 1 & $2,7 \%$ & 37 \\
\hline $\begin{array}{l}\text { Editor de wikis, } \\
\text { Wikispaces }\end{array}$ & 37 & $100 \%$ & o & - & o & - & o & - & - & o & - & 37 \\
\hline $\begin{array}{l}\text { Promedio total de } \\
\text { herramientas WEB } \\
2.0\end{array}$ & & $43,0 \%$ & & $37,6 \%$ & & $15,9 \%$ & & $3,3 \%$ & o\% & & $48,8 \%$ & $100 \%$ \\
\hline
\end{tabular}

Tabla 8 - Dominio de las herramientas TIC, Web 2.o por parte de los docentes de la UNAE, Ecuador, período 2019-2020.

Asimismo, de la Web 2.0, el 81,08\% de los 37 profesores del estudio afirman no tener dominio sobre los editores de video, el 67,57\% tampoco dominan las actividades educativas en las redes sociales como Facebook, Twitter, Instagram, el 56,76\%, no gestionan los blogs educativos por Blogger, WordPress, o las videoconferencias por Skype, Zoom, Adobe connect, el 59,46\% no tienen dominio de la gestión de herramientas de trabajo en grupo por Google Docs. El 94,59\% no domina la elaboración y edición de páginas web como Google-Sites, Wix y el 100\% no conoce o tiene dominio nulo de editor de Wiki como Wikispaces (ver tabla 8).

Por otro lado, ninguno de los docentes encuestados se catalogó como usuario muy avanzado o poseer conocimiento muy avanzado en el domino de las TIC con plataforma 
web 2.o. El 45,95\% y el 40,54\% tienen un dominio intermedio y básico, respectivamente, en la gestión de plataformas educativas como Moodle y Edmodo, mientras que el $45,95 \%$ tiene un dominio intermedio de video chat y aulas virtuales. El dominio de las herramientas web 2.0 enumeradas muestra que el 58,86\% de los profesores no las dominan en promedio, sólo el 23,12\% tiene un dominio básico de las mismas y el 13,51\% un dominio intermedio. En proporción, hay un mayor dominio en promedio de las herramientas de la Web 1.0 con 37,61\%, en comparación con la Web 2.0, con 23,12\%.

En el mismo orden de ideas, se promedió el uso de las herramientas de las TIC, la web 1.0 y la web 2.0. Debido a que algunos profesores afirmaron que dominaban algunas herramientas en sus niveles, pero al momento de preguntar cómo lo utilizaban en el proceso de enseñanza-aprendizaje, algunos afirmaron que no lo hacían con frecuencia, obteniendo los siguientes resultados reflejados en el gráfico 1 sobre el uso de estas herramientas tecnológicas.

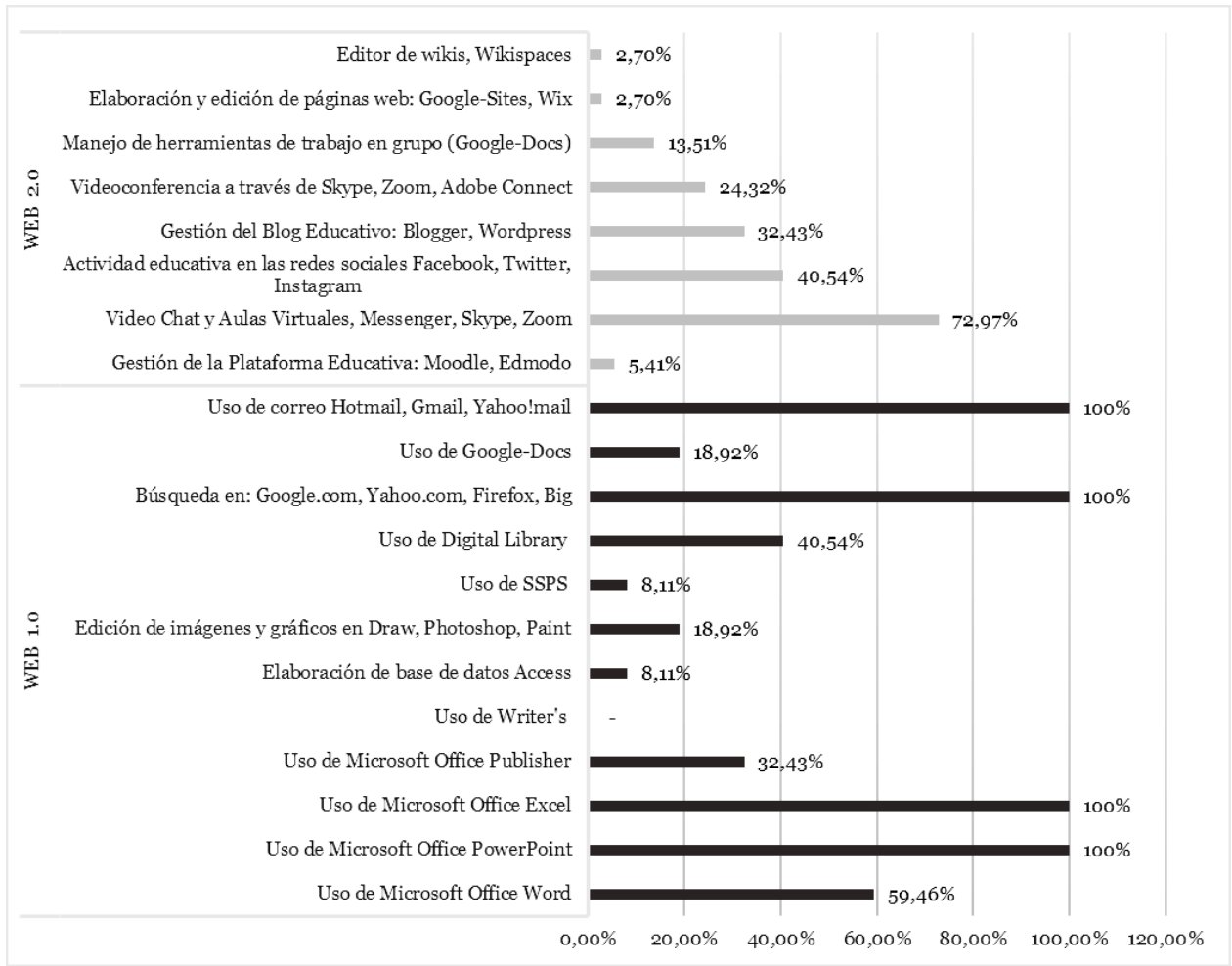

Figura 1 - Uso de herramientas TIC, web 1.o y web 2.0 por docentes de la UNAE, período 2019-2020

En cuanto a los profesores que afirmaban dominar el uso de las herramientas de Microsoft Office PowerPoint y Excel, los navegadores de búsqueda como Google.com, Yahoo.com, Firefox, Big y el correo electrónico a través de Hotmail, Gmail, Yahoo! mail también se denota que el 100\% de ese porcentaje utiliza o aplica estas herramientas para 
el proceso de enseñanza-aprendizaje. Sólo el 59,46\% de los profesores que afirmaron dominar el uso de las herramientas de Microsoft Office Word, el 32,43\% de Microsoft Office Publisher y el 40,54\% en la Gestión de Bibliotecas digitales, afirman que utilizan con frecuencia estas herramientas como soporte para sus clases (ver figura 1).

Asimismo, en cuanto al uso o aplicación de las herramientas TIC de la Web 2.0, los profesores que afirman dominar la gestión de plataformas educativas como Moodle y Edmodo, sólo el 72,97\% de ellos lo aplican en clase, de la misma manera el 40,54\% en video chat y aulas virtuales y el $32,43 \%$ de ellos con actividad educativa en las redes sociales Facebook, Twitter, Instagram.

Estos resultados ponen en perspectiva el dominio y uso de las herramientas TIC como gestión de las competencias digitales, en el gráfico 2 el uso total de éstas se promedia respecto a los profesores que afirmaron dominar algunas de ellas. Donde el uso de la web 1.o tiene un promedio de $48.87 \%$ y la web 2.0 un promedio de $21.62 \%$.

- El promedio total de uso en las clases de herramientas de la WEB 1.0

El promedio total de uso en las clases de herramientas de la WEB 2.0

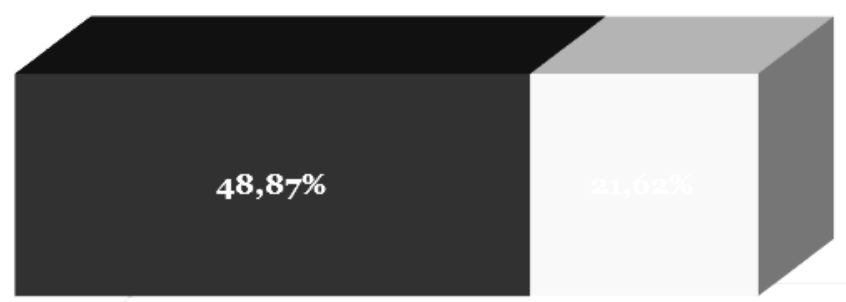

Figura 2 - Uso promedio de las herramientas TIC, Web 1.o y Web 2.0 por los profesores en el proceso de enseñanza-aprendizaje

En otras palabras, menos del 50\% utiliza las herramientas que dicen dominar con respecto a la web 1.0 y en el caso de la web 2.0, menos del 30\%. Esto indica que, aunque los profesores dominan algunas herramientas de las TIC, muchos de ellos no las aplican en el proceso de enseñanza-aprendizaje (ver figura 2).

Asimismo, los resultados mostraron una tendencia media del 53,71\%, lo que representa que sólo algo más del 50\% de los profesores de posgrado de la Institución de Educación Superior estudiada tiene competencias tecnológicas o digitales y el uso o aplicación en el 
aula. Se puede observar en la figura 3 a través de las categorías evaluadas la síntesis de la gestión de las competencias digitales de los docentes.

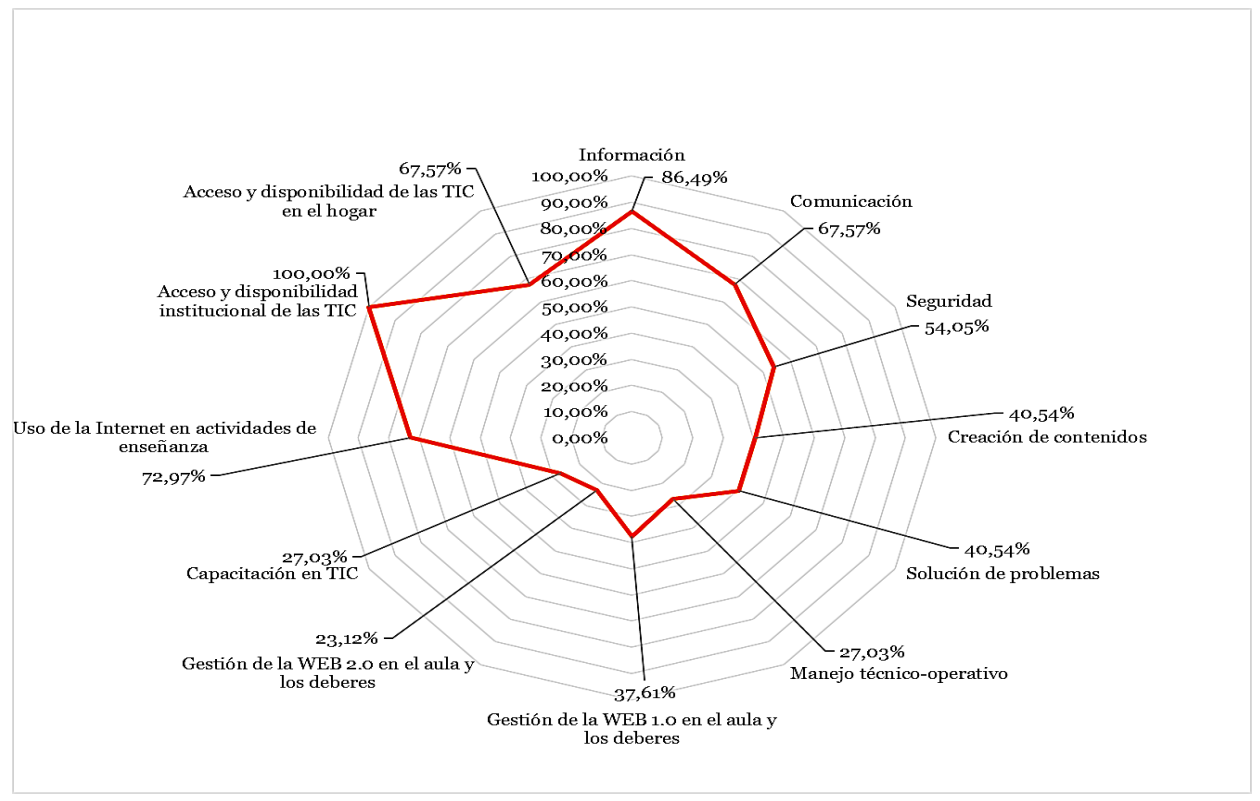

Figura 3 - Resumen de la situación de la gestión de las competencias digitales de los docentes de posgrado de una institución de educación superior en el Ecuador

En el estudio se presentaron conclusiones pertinentes, en las que las TIC son utilizadas principalmente por los profesores para la comunicación, afirmando mediante los instrumentos aplicados que la institución de enseñanza superior tiene acceso a las tecnologías y la disposición del desarrollo y la gestión de las competencias digitales tanto en los profesores, como en los estudiantes y todo el personal.

Sin embargo, sólo el $67,57 \%$ de los docentes tiene acceso y disponibilidad de los medios tecnológicos en sus hogares, lo que representa una limitación, ya que parte del trabajo se comparte entre la institución y el hogar, otro de los hallazgos. fue la falta de capacitación en tecnología, con sólo 27. El 03\%, menos del 50\% de los docentes han recibido capacitación en el área por parte de la Institución, tienen deficiencias en la gestión técnico-operativa y dificultades para resolver problemas relacionados con la tecnología o la digitalización de tareas, es decir, un alto grado de desconocimiento de las diferentes herramientas de la Web 2.o para gestionar la resolución de problemas en los procesos de enseñanza-aprendizaje (ver figura 3).

Sin embargo, es evidente que existe un déficit en la gestión de las competencias digitales, donde la tecnología se utiliza en su mayor parte para la comunicación y la información; la revisión del correo electrónico y el uso de las redes sociales, la descarga de documentos. 
Pero el conocimiento y la aplicación de las diferentes herramientas como el apoyo en el aula o los deberes no es evidente o su uso es inferior al 50\%. Siendo el profesor líder en el proceso de enseñanza-aprendizaje, debe cumplir las condiciones necesarias para demostrar un conocimiento superior al de sus alumnos.

\section{Conclusiones y recomendaciones}

Los cambios que se están produciendo tienen consecuencias para la educación y denotan el surgimiento de un nuevo contexto global de aprendizaje, creando nuevas condiciones que ameritan prácticas educativas generadas por este nuevo contexto de las TIC, mientras que las habilidades digitales representan una necesidad para obtener mejores resultados en el aula y mejorar la capacidad de enfoque y eficiencia en el proceso de enseñanza-aprendizaje, atendiendo las demandas y la forma de interpretar la realidad de la actual generación de estudiantes.

Cada día se incorporan más herramientas y avances que pueden apoyar a brindar una mayor calidad en la enseñanza, los jóvenes universitarios demandan a la velocidad que interpretan la realidad, la incorporación de las TIC y la visibilidad de las competencias digitales por parte de los docentes, es una ventaja dentro del proceso educativo que reduce las brechas de comunicación y exclusión.

Por ello, la disponibilidad y el acceso a las tecnologías es fundamental que, junto con la familiarización e incorporación de las diferentes herramientas digitales que pueden contribuir a este fin, suponga la demostración de una mayor calidad educativa para la comunidad científica en general. Por otra parte, se puede concluir que los medios tecnológicos son aplicados por los docentes, como entes de aplicación didácticas o recursos digitales para sus actividades educativas, mas no como un principal medio de investigación.

\section{Referencias}

Agnes, M., \& Norwich, B. (2007). Triangulation and Theoretical Understanding, International Journal of Social Research Methodology, 1O(3), 211-226. DOI: $10.1080 / 13645570701541878$

Azulai, A., \& Rankin, J. (2012). Triangulation in Canadian doctoral dissertations on aging. International Journal of Multiple Research Approaches, 6(2), 125-140. DOI: $10.5172 / \mathrm{mra} .2012 .6 .2 .125$

Battro, A., \& Percival, D. (2017). La educación digital. Argentina: EMECE.

Cejas Martínez, M.F., Mendoza Velazco, D.J., Navarro Cejas, M., Rogel Villacis, J.L., \& Ortega Freire, Y.M. (2019). A Performance-Centred Competency-Based Approach to Quality University Teaching. Integratsiya obrazovaniya = Integration of Education, 23(3), 350-365. DOI: https://doi.org/10.15507/1991-9468.096.023.201903. 350-365 
Chiecher, A., \& Lorenzati, K. (2017). Estudiantes y tecnologías. Una visión desde la 'lente' de docentes universitarios. RIED. Revista Iberoamericana de Educación a Distancia, 2O(1), 261-282.

De Llano, J. (2013). Estudiantes digitales / Profesores analogicos: La Universidad como punto de encuentro. In Las TIC en la educación universitaria. Venezuela, Caracas: (AUSJAL) Asociación de Universidades Confiadas a la Compañía de Jesús en América Latina.

Gardner, H. (2011). Frames of Mind: The Theory of Multiple Intelligences. Nueva York: Basic Books.

Lozada, B. (2019). Teoría de las inteligencias múltiples, el rol del docente y las aplicaciones móviles, triada estratégica de aprendizaje en la Educación Superior Universitaria. In 1er. Congreso Internacional y 2do. Congreso Nacional de Ciencia, Tecnología y Empresa. Tendiendo redes del conocimiento. Venezuela: Universidad Arturo Michelena.

Makewa, L., Ngussa, B., \& Kuboja, J. (2018). Technology-Supported Teaching and Research Methods for Educators. Spain: IGI Global.

Mendoza, D., La Madriz, J., López, M., \& Ramón, V. (2018). Research Competencies of Higher-Education Teaching Staff Based on Emotional Intelligence. Mediterranean Journal Of Social Sciences, 9(5), 41. Doi: 10.2478/mjss-2018-0137

Mendoza Velazco, D., Cejas Martínez, M., Navarro Cejas, M, Vega Falcón, V. \& Albán Yánez, C. (2019a). Moodle Research Software: Emotional Context in Ecuadorian Higher Education. International Journal of Engineering Research and Technology, 12(9), 1491-1500.

Mendoza, D., Abrigo, I., Romero, J., Cueva, F. \& Cejas, M. (2019b). The formative research of ecuadorian university teaching staff. Problems of education in the $21^{\text {st }}$ century, $77(3)$, 364-378.

Pedró, F. (2016). Hacia un uso pedagógico efectivo de la tecnología en el aula: ¿cómo mejorar las competencias docentes? (ONU-UNESCO, Ed.) Entornos digitales y políticas educativas. Dilemas y certezas, 245-267.

Pérez, J. (2013). Enseñanza 2.0: Uso de las redes sociales en las prácticas docentes. Las TIC en la educación universitaria, 38, 36-41.

Rendón, M., Villasís, M. \& Miranda, M. (2016). Estadística descriptiva. Rev Alerg Mex, 63(4), 397-407.

Spante, M., Sofkova, S., Lundin, M., \& Algers, A. (2018). Digital competence and digital literacy in higher education research: Systematic review of concept use. Cogent Education, 5(1). DOI: 10.1080/2331186X.2018.1519143

Yanez, S., Martinez, M., Mendoza, D., Lucano, S., Sanchez, H., \& Barros, R. (2019). The Solitary and Hatred within Social Media. Academic Journal of Interdisciplinary Studies, 8(3), 71. 
UNESCO (2013). Las TICs en la educación. (ONU, Ed.) Enfoque estrátegico sobre las TICS en Educación en América Latina y el Caribe.

Wright, S., Noble, R., \& Bailer, A. (2007). Equal-precision allocations and other constraints in stratified random sampling. Journal of Statistical Computation and Simulation, 77(12), 1081-1089. DOI: 10.1080/10629360600897191

Zuñiga, J. (2016). Las competencias digitales en el perfil universitario: El caso de la Facultad de Pedagogía de la Universidad Veracruzana. Mexico: Universidad Veracruzana. 